This item was submitted to Loughborough's Research Repository by the author.

Items in Figshare are protected by copyright, with all rights reserved, unless otherwise indicated.

\title{
Entanglement entropy of electronic excitations
}

PLEASE CITE THE PUBLISHED VERSION

https://doi.org/10.1063/1.4949535

PUBLISHER

AIP

VERSION

VoR (Version of Record)

\section{PUBLISHER STATEMENT}

This work is made available according to the conditions of the Creative Commons Attribution-NonCommercialNoDerivatives 4.0 International (CC BY-NC-ND 4.0) licence. Full details of this licence are available at: https://creativecommons.org/licenses/by-nc-nd/4.0/

\section{LICENCE}

CC BY-NC-ND 4.0

\section{REPOSITORY RECORD}

Plasser, Felix. 2019. "Entanglement Entropy of Electronic Excitations”. figshare.

https://hdl.handle.net/2134/32124. 


\title{
Entanglement entropy of electronic excitations
}

\author{
Felix Plassera) \\ Institute for Theoretical Chemistry, Faculty of Chemistry, University of Vienna, Währingerstr. 17, \\ 1090 Vienna, Austria
}

(Received 4 March 2016; accepted 2 May 2016; published online 18 May 2016)

\begin{abstract}
A new perspective into correlation effects in electronically excited states is provided through quantum information theory. The entanglement between the electron and hole quasiparticles is examined, and it is shown that the related entanglement entropy can be computed from the eigenvalue spectrum of the well-known natural transition orbital (NTO) decomposition. Non-vanishing entanglement is obtained whenever more than one NTO pair is involved, i.e., in the case of a multiconfigurational or collective excitation. An important implication is that in the case of entanglement it is not possible to gain a complete description of the state character from the orbitals alone, but more specific analysis methods are required to decode the mutual information between the electron and hole. Moreover, the newly introduced number of entangled states is an important property by itself giving information about excitonic structure. The utility of the formalism is illustrated in the cases of the excited states of two interacting ethylene molecules, the conjugated polymer para-phenylene vinylene, and the naphthalene molecule. Published by AIP Publishing. [http://dx.doi.org/10.1063/1.4949535]
\end{abstract}

\section{INTRODUCTION}

The computational description of electronic excitation processes has become a common task in quantum chemistry, and a wide variety of methods have been developed for this purpose. ${ }^{1-4}$ The wavefunctions produced in such calculations are usually described in terms of the canonical orbitals or by specific visualization protocols ${ }^{5}$ such as the natural orbitals, ${ }^{6}$ the natural transition orbitals (NTOs), ${ }^{7,8}$ and the attachment/detachment densities. ${ }^{9}$ While these techniques are convenient and intuitive, there is no fundamental reason why it should be even possible to represent electronic excitations by such one-particle functions. Indeed a number of cases are known where standard visualization techniques are insufficient and specialized protocols have been developed to quantify diverse specific properties such as excitonic effects, ${ }^{10-15}$ spatial correlation, ${ }^{16}$ collectivity, ${ }^{7,17,18}$ charge transfer, ${ }^{17,19-22}$ double excitation character, ${ }^{5,23}$ and orbital relaxation. ${ }^{24}$ In some cases these methods simply provide a quantitative description of phenomena that are also apparent otherwise while in other cases sophisticated analysis strategies are needed to even get a qualitative description of excited state characters, see, e.g., Refs. 10, 12, 13, and 17.

The analysis of excited state wavefunctions is particularly challenging in the cases of interacting chromophores and extended $\pi$-systems where dynamic charge transfer, charge resonance effects, and excitonic correlation come into play. This problem is most readily seen in the case of symmetric dimers where the differentiation between excitonic and charge resonance states is not possible by a visualization of the delocalized canonical orbitals alone but only by analyzing the signs of the individual configurations. ${ }^{13,17,25,26}$ In practice this means that special care has to be taken whenever interacting chromophores are present, as for example in

\footnotetext{
a)Electronic mail: felix.plasser@univie.ac.at
}

the case of DNA. ${ }^{27}$ Furthermore, in the case of interacting pentacene molecules, the issue of differentiating between locally excited and charge resonance character from an NTO analysis has even led to significant controversy. ${ }^{28-30}$ In the case of molecules with extended $\pi$ systems it has been understood for a while that non-standard analysis methods are particularly valuable, and these have been applied successfully by various groups. ${ }^{10-12,31-36}$ It has been our aim to supply a formalism that allows to analyze the wavefunctions of interacting chromophores and extended systems in a general quantum chemical context. Whereas our work was initially based on phenomenological reasoning, ${ }^{17}$ we have recently embedded our approach in a more solid formalism within exciton theory. ${ }^{5,13}$ The purpose of this work is to connect these analysis strategies to another well-established physical concept, the theory of quantum entanglement. ${ }^{37}$

Whereas the idea of quantum entanglement traces back to the early days of quantum mechanics, ${ }^{37}$ significant additional work has been done in more recent years. ${ }^{38-40}$ The phenomenon is most readily studied in a bipartite system, i.e., a quantum system composed of two subsystems. In this case the entanglement is quantified by the entanglement entropy. If the composite system is in a pure state this entanglement entropy is given by the von Neumann entropy of the subsystems

$$
S_{A \mid B}=-\operatorname{tr}\left[\widehat{\rho}_{A} \log _{2} \widehat{\rho}_{A}\right]=-\operatorname{tr}\left[\widehat{\rho}_{B} \log _{2} \widehat{\rho}_{B}\right],
$$

where $\widehat{\rho}_{A}$ and $\widehat{\rho}_{B}$ are the reduced density operators of the subsystems $A$ and $B \cdot{ }^{38,39,41,42}$ The value of $S_{A \mid B}$ quantifies the mutual information between the two subsystems, i.e., the amount of information that is encoded in the correlations between the subsystems rather than in the subsystems themselves. ${ }^{39}$ Aside from some general discussions about electron correlation, ${ }^{43,44}$ the above ideas have found their entry into quantum chemistry mainly in the context of the density matrix renormalization group theory, ${ }^{41,45}$ and the computation of orbital entanglement. ${ }^{42,46}$ The idea of the current work is 
to apply the same formalism to excited states. Whereas a similar approach has been considered in the specific case of quantum dot pairs, ${ }^{47}$ the more general utility of this approach in a quantum chemical context is discussed here. The focus of this work is an analysis of entanglement on a fundamental level. However, it should be pointed out that entanglement of electronic excitations has also been discussed in more applied works, e.g., in the context of photosynthesis ${ }^{48,49}$ and quantum computing. 47,50

\section{METHODS}

\section{A. Exciton wavefunctions}

The formalism presented here is based on the exciton picture of an electronic excitation process. Rather than analyzing the full many-body wavefunctions, an effective two-body exciton wavefunction $\chi_{\text {exc }}\left(r_{h}, r_{e}\right)$ is constructed describing the transition process from the ground to the excited state in terms of the hole $\left(r_{h}\right)$ and electron $\left(r_{e}\right)$ coordinates. This exciton wavefunction is simple enough to allow for a rather intuitive understanding of the excitation process, and it can be readily subjected to a detailed quantitative analysis. ${ }^{5,13,16}$ At the same time the information content of this two-body function clearly exceeds that of individual orbitals or densities.

The exciton wavefunction is usually expanded with respect to an orthonormal basis set of spatial orbitals $\left\{\phi_{p}\right\}$,

$$
\chi_{e x c}\left(r_{h}, r_{e}\right)=\sum_{p q} D_{p q} \phi_{p}\left(r_{h}\right) \phi_{q}\left(r_{e}\right) \text {. }
$$

In the case of an effective one-electron theory, such as configuration interaction with single excitations (CIS), timedependent density functional theory (TDDFT), or the BetheSalpeter equation, the electron-hole amplitudes $D_{p q}$ are usually directly equated with the response vector elements. ${ }^{12,51,52}$

We have argued that a natural way to obtain an exciton wavefunction from arbitrary many-electron wavefunctions is to use the 1-electron transition density matrix (1TDM) between the ground state and the excited state of interest. ${ }^{5,13}$ This can be understood by using Green's function theory, ${ }^{53}$ by using current operators, ${ }^{54}$ or by considering ${ }^{55}$ that the 1TDM presents the best pair function for constructing the excited state on top of the ground state. The advantage of using the 1TDM is that this is a well-defined quantity independent of the wavefunction model and, indeed, density matrix based approaches have been applied to compare results between different computational methods. ${ }^{24,56-58}$

Finally, two limitations of this approach should be pointed out. First, the analysis is only meaningful for states with dominant one-electron excitation character. Second, a clear interpretation of the results is only possible if the ground state possesses closed shell character. However, these cases certainly encompass a large fraction of the excited states of interest in organic molecules.

\section{B. Electron-hole entanglement}

It is seen in Eq. (2) that the exciton wavefunction is given as a linear combination of products of hole and electron orbitals. To emphasize this direct product nature, the nomenclature of Refs. 39 and 42 is applied and Eq. (2) is rewritten in the form

$$
\left|\chi_{\text {exc }}\right\rangle=\sum_{p q} D_{p q}\left|\phi_{p}^{H}\right\rangle \otimes\left|\phi_{q}^{E}\right\rangle .
$$

Using this nomenclature, the density operator of the exciton (describing a pure quantum state) is given as

$$
\begin{aligned}
\widehat{\rho}_{\text {exc }} & =\left|\chi_{\text {exc }}\right\rangle\left\langle\chi_{\text {exc }}\right| \\
& =\sum_{p q} \sum_{r s} D_{p q} D_{r s}\left|\phi_{p}^{H}\right\rangle\left\langle\phi_{r}^{H}|\otimes| \phi_{q}^{E}\right\rangle\left\langle\phi_{s}^{E}\right| .
\end{aligned}
$$

The reduced density operator of the electron subsystem is computed by taking the partial trace ${ }^{39,42}$ over the $H$ subsystem, which leads to the equation

$$
\widehat{\rho}_{E}=\operatorname{tr}_{H}\left[\widehat{\rho}_{e x c}\right]=\sum_{t}\left\langle\phi_{t}^{H}\left|\widehat{\rho}_{e x c}\right| \phi_{t}^{H}\right\rangle .
$$

Insertion of $\widehat{\rho}_{\text {exc }}$ from Eq. (4) and using the fact that the orbitals are orthonormal yields

$\widehat{\rho}_{E}=\sum_{q s} \sum_{t} D_{t q} D_{t s}\left|\phi_{q}^{E}\right\rangle\left\langle\phi_{s}^{E}\left|=\sum_{q s}\left(\mathbf{D}^{T} \mathbf{D}\right)_{q s}\right| \phi_{q}^{E}\right\rangle\left\langle\phi_{s}^{E}\right|$.

Here, the expression $\mathbf{D}^{T} \mathbf{D}$ is the well-known density matrix of the excess electron, as discussed, e.g., in Refs. 5, 59, and 60. In analogy to Eq. (6), the reduced hole density operator is given as

$$
\widehat{\rho}_{H}=\sum_{p r}\left(\mathbf{D D}^{T}\right)_{p r}\left|\phi_{p}^{H}\right\rangle\left\langle\phi_{r}^{H}\right| .
$$

The spectral representation of the two operators is obtained by diagonalizing the $\mathbf{D}^{T} \mathbf{D}$ and $\mathbf{D D}^{T}$ matrices

$$
\begin{aligned}
& \widehat{\rho}_{H}=\sum_{i} \lambda_{i}\left|\psi_{i}^{H}\right\rangle\left\langle\psi_{i}^{H}\right|, \\
& \widehat{\rho}_{E}=\sum_{i} \lambda_{i}\left|\tilde{\psi}_{i}^{E}\right\rangle\left\langle\tilde{\psi}_{i}^{E}\right|,
\end{aligned}
$$

where in both cases the same eigenvalues $\lambda_{i}$ are obtained. This formalism, which corresponds to a singular value decomposition of $\mathbf{D}$, is widely used in quantum chemistry $7,8,17,61-63$ and is usually termed the natural transition orbital (NTO) decomposition.

At this point it should be clarified that the orbital basis set $\left\{\phi_{p}\right\}$ appears only for technical reasons and none of the following results depend on the choice of the $\phi_{p}$ orbitals as long as they form a complete orthonormal basis with respect to the wavefunctions analyzed. Indeed, it is possible to construct the electron and hole densities ${ }^{5}$ and therefore the $\widehat{\rho}_{E}$ and $\widehat{\rho}_{H}$ operators directly in coordinate space. The NTOs are well defined as the eigenfunctions of these operators even for electronic structure methods that are not based on orbitals.

Given the electron and hole density operators, it is possible to define the entanglement entropy between the electron and hole subsystems ${ }^{47}$ in analogy to Eq. (1),

$$
S_{H \mid E}=-\operatorname{tr}\left[\widehat{\rho}_{E} \log _{2} \widehat{\rho}_{E}\right]=-\operatorname{tr}\left[\widehat{\rho}_{H} \log _{2} \widehat{\rho}_{H}\right] .
$$

In the spectral representation of Eqs. (8) and (9) this yields the simple formula 


$$
S_{H \mid E}=-\sum_{i} \lambda_{i} \log _{2} \lambda_{i},
$$

that can be evaluated directly from the NTO eigenvalues. Aside from the entropy, we define the effective number of entangled states as

$$
Z_{H E}=2^{S_{H \mid E}}=1 / \prod_{i} \lambda_{i}^{\lambda_{i}},
$$

which is arguably a more intuitive quantity as will be discussed below, and Eq. (12) will be used as the main working equation to quantify entanglement.

The $Z_{H E}$ value possesses a simple interpretation under the assumption that $m$ configurations contribute with equal weights $^{64}$

$$
\begin{gathered}
\lambda_{1}=\lambda_{2}=\cdots=\lambda_{m}=1 / m, \\
\lambda_{m+1}=\lambda_{m+2}=\cdots=0 .
\end{gathered}
$$

In this case it holds that $Z_{H E}=m$ is just the number of configurations involved.

From a numerical point of view the number of entangled states $Z_{H E}$ is related to the collectivity number

$$
\kappa=\frac{\left(\operatorname{tr} \mathbf{D}^{\mathrm{T}} \mathbf{D}\right)^{2}}{\operatorname{tr}\left(\mathbf{D}^{\mathrm{T}} \mathbf{D}\right)^{2}},
$$

which was introduced by Luzanov with the main motivation that it is a lower bound to the matrix rank of $\mathbf{D}^{7,19}$ An equivalent formula ${ }^{65}$

$$
\kappa=\mathrm{PR}_{\mathrm{NTO}}=\frac{\left(\sum_{i} \lambda_{i}\right)^{2}}{\sum_{i} \lambda_{i}^{2}}
$$

was introduced with the idea of counting the number of NTO configurations as a participation ratio. ${ }^{5,17}$ In the case of equal NTO amplitudes [Eq. (13)] the collectivity number and the number of entangled states both give $Z_{H E}=\kappa=m$, while for more realistic cases we observe that $Z_{H E}$ is generally somewhat larger than $\kappa$.

\section{Spatial analysis of exciton wavefunctions}

In contrast to the analysis of Sec. II B, which is entirely based on the NTO eigenvalue spectrum, we have previously been concerned with an analysis of the spatial distribution of exciton wavefunctions. ${ }^{13,16,17}$ Some of these ideas will be shortly reviewed here. One instructive analysis method is to divide the system into fragments and compute a partial integral over $\chi_{\text {exc }}$ where the hole is restricted to one molecular fragment $A$ while the electron is restricted to fragment $B$ yielding the charge transfer number ${ }^{17,19}$

$$
\Omega_{A B}=\int_{A} \mathrm{~d} r_{h} \int_{B} \mathrm{~d} r_{e} \chi_{e x c}\left(r_{h}, r_{e}\right)^{2} .
$$

Here, a diagonal element $\Omega_{A A}$ quantifies the weight of local excitations on $A$ whereas an off-diagonal element $\Omega_{A B}$ quantifies the charge transfer from fragment $A$ to $B$. The total amount of charge separation, which will be used below, is given as the sum

$$
\omega_{C T}=\frac{1}{\Omega} \sum_{A} \sum_{B \neq A} \Omega_{A B}
$$

over all off-diagonal charge transfer numbers. Here, $\Omega=$ $\left\langle\chi_{\text {exc }} \mid \chi_{\text {exc }}\right\rangle$ is the squared norm of the exciton wavefunction.

We have recently extended this methodology to the computation of operator expectation values with respect to the exciton wavefunction ${ }^{13,16}$

$$
\langle\hat{O}\rangle=\frac{\left\langle\chi_{e x c}|\hat{O}| \chi_{e x c}\right\rangle}{\left\langle\chi_{e x c} \mid \chi_{e x c}\right\rangle},
$$

which can be readily evaluated for any operator whose matrix elements in the underlying orbital representation are available. ${ }^{13}$ One such expectation value is the root-meansquare (RMS) electron-hole separation

$$
d_{e x c}=\sqrt{\left\langle\left|r_{e}-r_{h}\right|^{2}\right\rangle},
$$

which is interpreted as the exciton size. ${ }^{13}$ As discussed previously, the $d_{\text {exc }}$ value does not only cover static CT that can be deduced directly from the orbitals but it also includes dynamic effects that derive from the interactions between different NTO transitions. ${ }^{13,16}$

Long-range correlation effects between the electron and hole quasiparticles are computed in analogy to the Pearson correlation coefficient

$$
R_{e h}=\frac{\left\langle r_{h} \cdot r_{e}\right\rangle-\left\langle r_{h}\right\rangle \cdot\left\langle r_{e}\right\rangle}{\sigma_{h} \sigma_{e}},
$$

where $\sigma_{h}$ and $\sigma_{e}$ are the RMS sizes of the hole and electron distributions. ${ }^{16}$ This value ranges from -1 (perfect anti-correlation) through 0 (no correlation) to 1 (perfect correlation). Positive values of $R_{e h}$ are associated to bound excitons, whereas negative values are related to effective electron-hole repulsion. The $R_{e h}$ value is concerned with correlations in the electron-hole picture ${ }^{53}$ of the excitation. While a full comparison to the standard electron correlation picture in quantum chemistry is out of the scope of this work, it should be pointed out that the concepts are related. It can be worked out analytically that a non-vanishing $R_{e h}$ value can only be obtained for multiconfigurational excited states ${ }^{16}$ and it will be shown below that there is indeed a semi-quantitative correspondence between the number of entangled states (as a measure for multiconfigurational character) and the electronhole correlation coefficient.

\section{Implementation and computational details}

The $S_{H \mid E}$ and $Z_{H E}$ quantities are readily available if NTOs are already computed and we have included them together with our previous NTO implementation ${ }^{5,17}$ in Q-CHem 4.3 (accessed with the state_analysis and nto_pairs keywords). ${ }^{66}$ An additional implementation is provided within the THEODORE 1.2 program package (using prop_list=['S_HE' , 'Z_HE'] $),{ }^{67}$ which contains interfaces to a wide range of quantum chemistry codes using (in part) the cCLIB library. ${ }^{68}$ An implementation of our wavefunction analysis tools within Molcas 8.1 is currently in progress. ${ }^{69}$

Excited state computations on the ethylene dimer were performed by means of the algebraic diagrammatic construction method to second order $\operatorname{ADC}(2)^{70,71}$ in connection with the $\mathrm{SV}(\mathrm{P})$ basis set. ${ }^{72}$ TDDFT computations were performed 
in the Tamm-Dancoff approximation using the CAM-B3LYP functional ${ }^{73-75}$ and the SVP basis set. Naphthalene was optimized in the ground state at the DFT/B3LYP level while in the case of the PPV oligomers the geometries were taken from Ref. 76. All computations were performed with a developmental version of the Q-CHEM 4.3 program package. ${ }^{66,77}$ Orbital visualizations were carried out in JMOL $14 .{ }^{78}$

\section{RESULTS AND DISCUSSION}

\section{A. Direct product wavefunction}

To understand the meaning of the entanglement entropy, it is beneficial to start with the very simple case where the spatial exciton wavefunction can be described as a product of two orbitals

$$
\chi_{\text {exc }}\left(r_{h}, r_{e}\right)=\psi^{H}\left(r_{h}\right) \tilde{\psi}^{E}\left(r_{e}\right),
$$

i.e., the case where only one NTO pair contributes to the excitation $\left(\lambda_{1}=1\right.$ and $\left.\lambda_{2}=\lambda_{3}=\cdots=0\right)$. In this case the density operator describes a direct product state, the reduced density operators are pure states in the subsystems and the mutual information vanishes, yielding $S_{H \mid E}=0$ and $Z_{H E}=1$. Considering that there is no mutual information between the electron and hole subsystems, it suffices to inspect the two orbitals $\psi^{H}$ and $\tilde{\psi}^{E}$ independently to have a complete description of the excitation process. The direct product nature of the excited state can be apparent in the canonical orbital representation, e.g., in the case of a simple excitation from the highest occupied MO (HOMO) to the lowest unoccupied MO (LUMO), whereas in other cases this is only seen after the NTO transformation. The latter case has been termed false collectivity. ${ }^{79}$ It can be worked out that in the case of a direct product wavefunction the correlation coefficient $R_{e h}$ vanishes. ${ }^{16}$ Electron-hole correlation and entanglement are thus only present for multiconfigurational wavefunctions, in agreement with the general notion of equating multiconfigurational nature with correlation.

\section{B. Dimer model}

The model of two weakly interacting chromophores serves as a valuable tool to elucidate some non-intuitive properties of electronic excitations. ${ }^{13,17,25}$ Here, I want to revisit this type of system in a quantum bit (qubit) notation (see, e.g., Ref. 39). For this purpose, the molecules are indexed 0 and 1, and the electron and hole are marked by superscripts $E$ and $H$. This two-qubit system allows to construct four excited states, two locally excited ones $\left|0^{H}, 0^{E}\right\rangle$ and $\left|1^{H}, 1^{E}\right\rangle$, as well as two charge transfer states $\left|0^{H}, 1^{E}\right\rangle$ and $\left|1^{H}, 0^{E}\right\rangle$. Using this basis, two Frenkel exciton states

$$
\left|\Phi_{ \pm}\right\rangle=2^{-1 / 2}\left(\left|0^{H}, 0^{E}\right\rangle \pm\left|1^{H}, 1^{E}\right\rangle\right)
$$

are constructed as linear combinations of the local states while the charge resonance states

$$
\left|\Psi_{ \pm}\right\rangle=2^{-1 / 2}\left(\left|0^{H}, 1^{E}\right\rangle \pm\left|1^{H}, 0^{E}\right\rangle\right)
$$

are linear combinations of the charge transfer states. Incidentally, the excitonic and charge resonance states as discussed here and in a number of other works ${ }^{13,17,25,26}$ possess the same form as the so called Bell states, which are the maximally entangled states of two qubits. ${ }^{39}$ The presence of entanglement, i.e., a quantum effect without a classical analogue, sheds new light on our previous observation ${ }^{13,17}$ that an understanding of state characters in such cases is challenging. The problem is seen more readily if the reduced density operators are computed. These are equivalent in all four cases ${ }^{39}$ giving

$$
\begin{aligned}
& \widehat{\rho}_{H}=1 / 2\left(\left|0^{H}\right\rangle\left\langle 0^{H}|+| 1^{H}\right\rangle\left\langle 1^{H}\right|\right), \\
& \widehat{\rho}_{E}=1 / 2\left(\left|0^{E}\right\rangle\left\langle 0^{E}|+| 1^{E}\right\rangle\left\langle 1^{E}\right|\right) .
\end{aligned}
$$

Consequently, an analysis in terms of independent electron and hole densities does not provide any information as to which one of the four states $\left|\Phi_{+}\right\rangle,\left|\Phi_{-}\right\rangle,\left|\Psi_{+}\right\rangle,\left|\Psi_{-}\right\rangle$ was initially present. In practical cases the situation is somewhat improved as the NTO decomposition preserves some of the mutual information by connecting the individual hole and electron NTOs. However, recovering the phase information, which is necessary to differentiate between excitonic and charge resonance states in the case of delocalized orbitals,,${ }^{17,25}$ is more challenging and requires specialized tools.

In the above case [Eqs. (24) and (25)], there are two NTO pairs with equal weights $\left(\lambda_{1}=\lambda_{2}=1 / 2\right)$ and consequently $S_{H \mid E}=1, Z_{H E}=2$. The fact that two nonvanishing NTO eigenvalues are present for excited states of two interacting chromophores was pointed out initially by Mayer. ${ }^{80}$ Subsequently, we argued based on Ref. 81 that this reflects correlation between the electron and hole quasiparticles. ${ }^{16,17}$ Here, a new interpretation is added: the specific form of the states reflects entanglement.

As a practical example, two interacting ethylene molecules are studied and the first singlet excited state is computed at various intermolecular separations. The primary NTOs for the cases of $6.0 \AA$ and $3.5 \AA$ intermolecular separation are shown in Figures 1 and 2. The relevant transitions occur between $\pi$ and $\pi^{*}$ orbitals. These possess similar shapes for the $6.0 \AA$ and $3.5 \AA$ cases and a superficial analysis might lead to the conclusion that the excited states

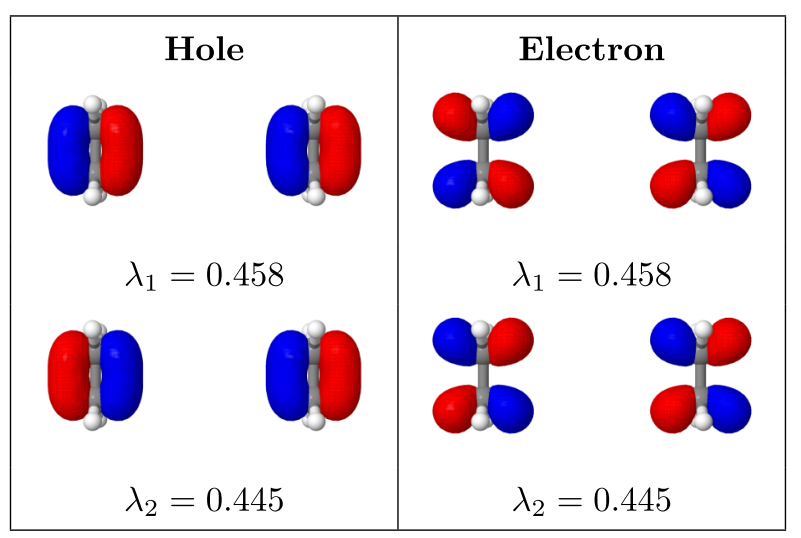

FIG. 1. Natural transition orbitals (isovalue 0.05) of the first excited state of two interacting ethylene molecules at $6.0 \AA$ intermolecular separation computed at the ADC(2)/SV(P) level of theory. 


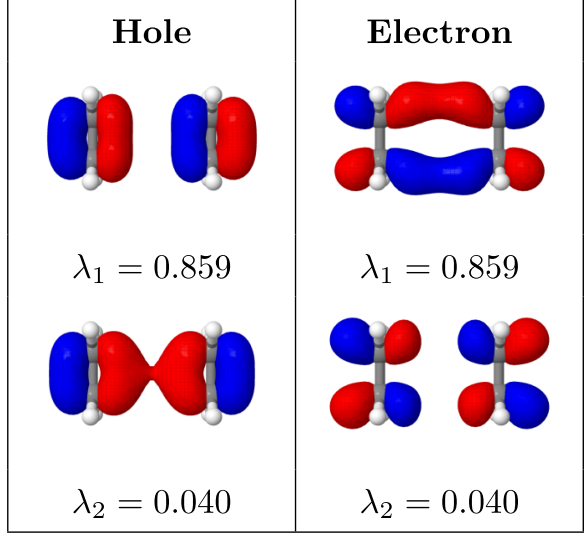

FIG. 2. Natural transition orbitals (isovalue 0.05) of the first excited state of two interacting ethylene molecules at $3.5 \AA$ intermolecular separation computed at the $\mathrm{ADC}(2) / \mathrm{SV}(\mathrm{P})$ level of theory.

are similar and possess locally excited character in both cases. However, once the eigenvalue spectrum is taken into account, this assessment changes dramatically. At $6.0 \AA$ separation there are two transitions with about equal weights $\left(\lambda_{1} \approx \lambda_{2} \approx\right.$ 0.45 ) whereas in the case of $3.5 \AA$ separation there is only one strongly dominant transition $\left(\lambda_{1}=0.86\right)$. Clearly, there are significant qualitative differences in wavefunction character between these two states. The above discussion helps in the interpretation of these results. Only the case at $6.0 \AA$ separation exhibits two NTO transitions with equal weights as required for a Frenkel exciton state [Eq. (22)]. By contrast, the single dominant NTO transition at $3.5 \AA$ separation is an indication of admixture of charge resonance character, as will be discussed in more detail below. This charge resonance character is not visible with respect to the delocalized canonical orbitals (Figure 2) but would be apparent, e.g., for fragment-localized ${ }^{15}$ orbitals.

In order to study the system in a quantitative manner, the energy of the first excited state is plotted with respect to the intermolecular separation in Figure 3(a). Starting from the right (large separations) the energy is almost constant until about $5.0 \AA$ separation and strongly decreases afterwards reaching an exciplex type minimum at around $2.2 \AA$ separation. It should be noted that below $2.5 \AA$ separation also a doubly excited state comes into play and photodimerization is initiated. ${ }^{82}$ However, until that point the $\operatorname{ADC}(2)$ method, as chosen here, is certainly expected to produce accurate wavefunctions. To monitor the change in wavefunction character, we use the number of entangled states $Z_{H E}$ as defined in Eq. (12) (Figure 3(b), black circles). At large separations one obtains $Z_{H E}=2.8$. This large value derives from the fact that two configurations show some major involvement in the excitation process as shown in Fig. 1. Once the molecules move close together, a dramatic decrease in $Z_{H E}$ is observed. At $3.5 \AA$ separation (cf. Figure 2) this value is 1.5 and it converges to about 1.2 at lower separations. The crucial finding of Figure 3 is that the $Z_{H E}$ value goes more or less parallel to the energy. In both cases the onset of the decrease happens at $5.0 \AA$, then there is a steep decay, and a flatter profile around the exciplex minimum. This underlines that entanglement, as discussed here, is a decisive property of

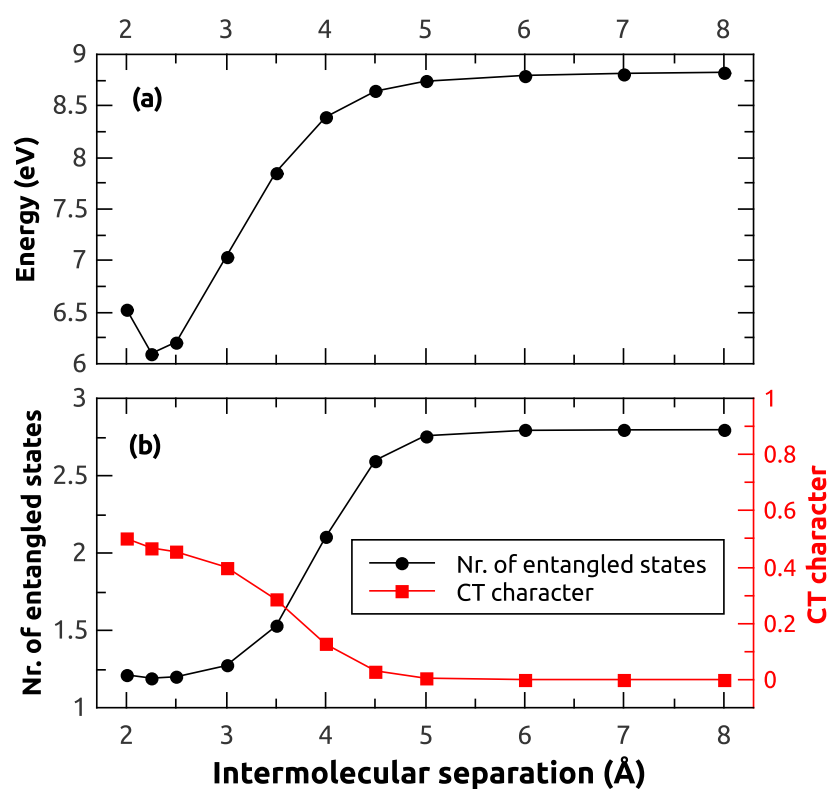

FIG. 3. Analysis of the first excited singlet state of two interacting ethylene molecules with varying intermolecular separation computed at the $\mathrm{ADC}(2) / \mathrm{SV}(\mathrm{P})$ level of theory: (a) energy relative to the ground state at large separation, (b) number of entangled states $\left(Z_{H E}\right)$ and $C T$ character $\left(\omega_{C T}\right)$.

the wavefunction with severe implications on the energetics of the system.

The power of the previous analysis is that it is only concerned with the NTO eigenvalue spectrum and does not require any more specific analysis of the wavefunctions. However, it is also interesting to apply our previous spatial analysis protocols to this system and investigate the CT character of the system. Whereas there is no net charge transfer in this symmetric system it is possible that the electron and hole are separated in a dynamic fashion, leading to so called charge resonance states. The amount of charge resonance admixture is quantified by the $\omega_{C T}$ value [Eq. (17)] as shown in Figure 3(b). For large separations $\omega_{C T}$ amounts to 0 showing that the state is a pure Frenkel exciton. At lower separations, starting at about $5.0 \AA$, a steady increase occurs and the value converges to about $\omega_{C T}=0.5$, i.e., an even mixture between Frenkel and CT character. A closer inspection of Figure 3(b) shows that the CT character behaves as a mirror image to the number of entangled states, i.e., the loss in entanglement as the molecules move together is accompanied by an increase in CT admixture. The impressive feature of this analysis is that two entirely different analysis strategies, an analysis of the eigenvalue spectrum and a population analysis, yield consistent trends,

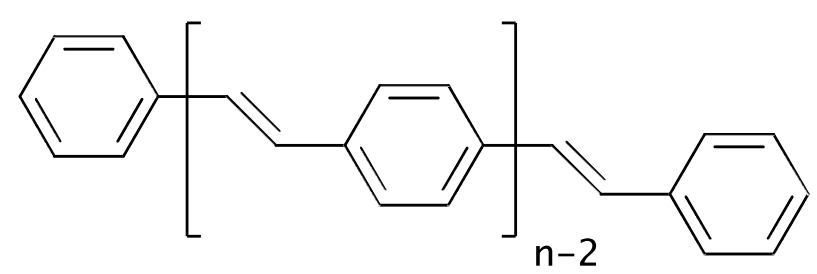

FIG. 4. Molecular structure of the PPV system studied here for varying number of phenyl rings $n=2, \ldots, 8$. 


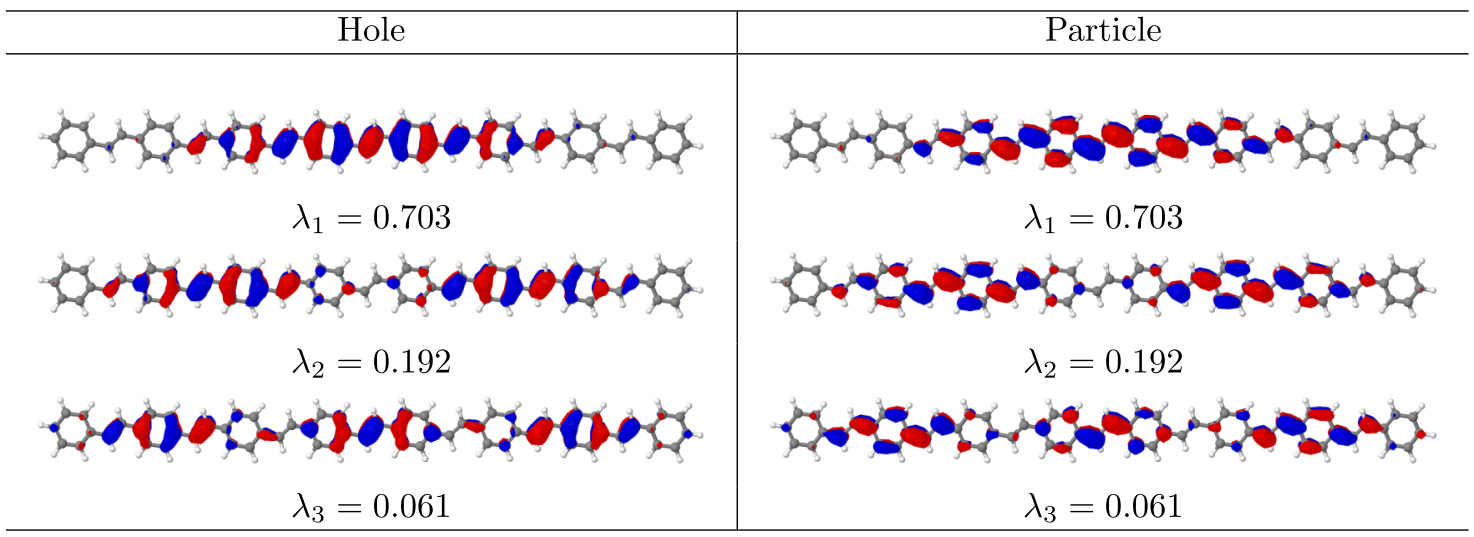

FIG. 5. NTOs of the $S_{1}$ state of $(\mathrm{PV})_{7} \mathrm{P}$ computed at the CAM-B3LYP/SVP level of theory. All NTOs with $\lambda_{i}>0.03$ are plotted, cutoff value: 0.02 .

which furthermore reflect the trends of the total energy of the system. The picture drawn above is generally consistent with our previous analyses of the naphthalene, pyridine, and adenine dimers ${ }^{13,17,83}$ and of a system of six interacting neon atoms. $^{24}$

\section{Conjugated polymers}

The conjugated polymer poly(para-phenylene vinylene) (PPV, Figure 4) is chosen as a more challenging system for testing the presented formalism, and its excited states for varying chain length are investigated. We have recently studied this system in great detail ${ }^{36,76}$ and shall restrict ourselves to some exemplary analyses here. The discussion is started with the $S_{1}$ and $T_{1}$ states of $(\mathrm{PV})_{7} \mathrm{P}$, i.e., the oligomer containing eight phenyl rings. For this system, we want to discuss the analysis of excited state character in some detail using a hierarchy of three strategies: (i) a depiction of only the dominant NTO transition, (ii) an analysis of all NTOs and computation of the entanglement, and (iii) specific more involved multipole analysis protocols. Option (i) is a common and straightforward application of the NTO analysis, which in simple cases provides all the information of interest. The dominant NTO pair of the $S_{1}$ state is shown in the first line of Figure 5. The hole and particle NTOs possess $\pi$ and $\pi^{*}$ character and are both localized on the central four phenyl rings with major contributions on the central two. Interestingly the dominant NTO pair for the $T_{1}$ state (Figure 6) is practically identical to the one of the $S_{1}$ state, and at this stage one might presume that, aside from spin-symmetry, the $S_{1}$ and $T_{1}$ wavefunctions are identical. Application of strategy (ii) significantly alters the viewpoint. Whereas the shapes of the secondary NTOs between the $S_{1}$ and $T_{1}$ states are quite similar, their amplitudes differ significantly. The question arises whether the differences in NTO amplitudes are a type of numerical noise or whether they can be assigned a physical meaning. For this purpose we suggest using the number of entangled states $Z_{H E}$ [Eq. (12)] as a well-defined descriptor to quantify the physical role of the secondary contributions. In the case of the singlet there are $Z_{H E}=2.6$ states involved, i.e., there are two configurations with significant weights and some additional smaller contributions as shown in Figure 5. For the triplet this number is enhanced to 4.1 as more configurations are involved (Figure 6). From this analysis it is already clear that the $S_{1}$ and $T_{1}$ wavefunctions differ significantly. Furthermore, the enhanced $Z_{H E}$ values imply

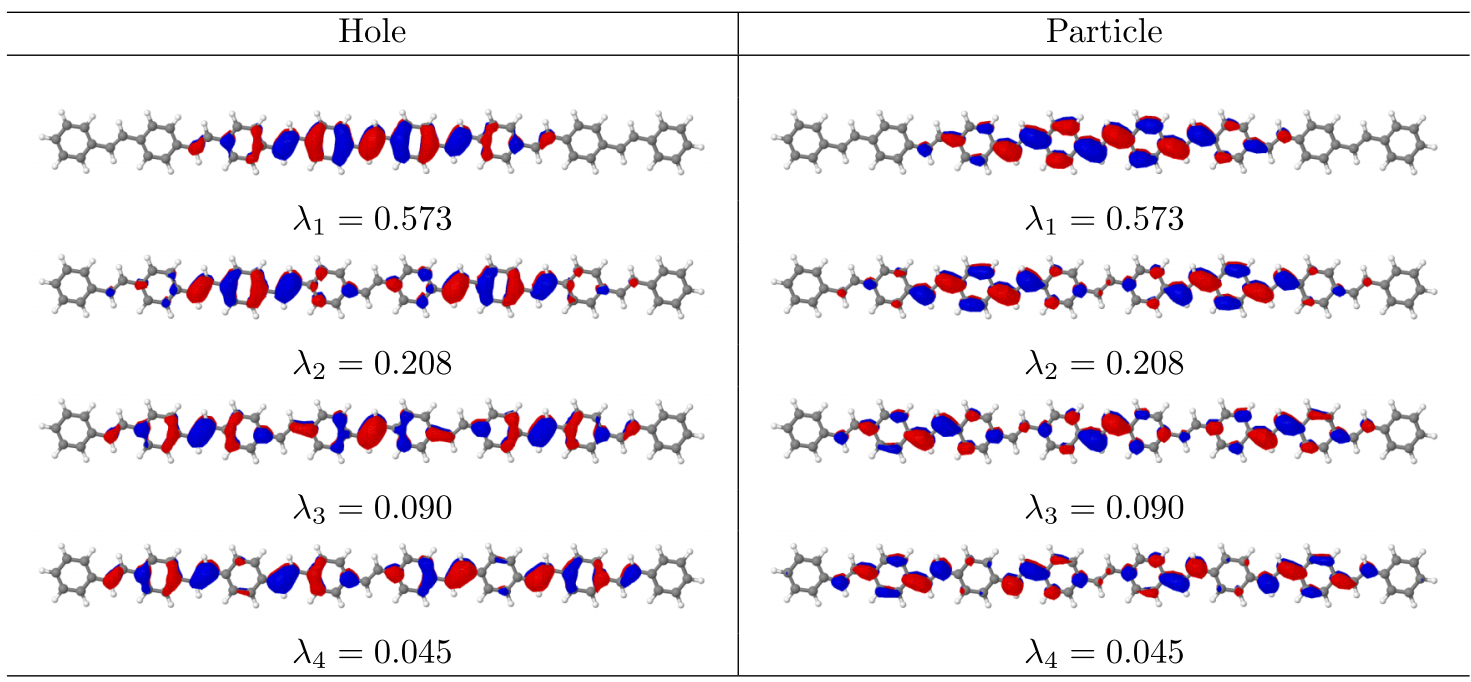

FIG. 6. NTOs of the $T_{1}$ state of (PV) $)_{7} \mathrm{P}$ computed at the CAM-B3LYP/SVP level of theory. All NTOs with $\lambda_{i}>0.03$ are plotted, cutoff value: 0.02 . 
that orbital depictions do not show all information of interest about this state but that there are also electron-hole correlation effects hidden as mutual information. Steps (i) and (ii), performed so far, are a quite general procedure, whereas the next step (iii) amounts to specifically decoding the mutual information. In general this third step is more involved and has to be tailored to the system of interest. We have recently developed such analysis protocols for conjugated organic polymers $^{36,76}$ and shall restrict ourselves to two of the most instructive quantities: the exciton size and the correlation coefficient. First, the exciton size $d_{\text {exc }}$ [Eq. (19)] is computed describing the averaged distance between the electron and hole quasiparticles. This value amounts to $6.9 \AA$ and $5.3 \AA$ for $S_{1}$ and $T_{1}$, respectively. The triplet possesses the smaller exciton size, i.e., it is more tightly bound. This result, which is in agreement with general exciton theory, ${ }^{31}$ follows directly from application of Eq. (19) whereas it is virtually impossible to deduce from a pictorial NTO analysis. Interestingly, the exciton sizes are on the order of the length of just one PV unit (5.3 $\AA$ ). These small values are at first surprising considering that the NTOs are quite delocalized as seen in Figures 5 and 6. The reason for this discrepancy is long-range correlation, a property that can be monitored by the recently introduced electron-hole correlation coefficient $R_{e h}$ [Eq. (20)]. ${ }^{16}$ In the case of the singlet, the $R_{e h}$ value is 0.78 and for the triplet one obtains $R_{e h}=0.85$.

As a next step, the above properties will be investigated for PPV oligomers of varying chain lengths, going from PVP to $(\mathrm{PV})_{7} \mathrm{P}$. In Figure 7 (a) the exciton size of the lowest singlet and triplet states is plotted against the number of phenyl rings. The exciton size increases at first but levels off quickly, in agreement with previous studies. ${ }^{36,84}$ The values converge to about $7 \AA$ and $5.5 \AA$ for the singlet and triplet, respectively. This exciton size limit derives from long-range correlation effects, which are again monitored using the electron-hole correlation coefficient, as shown in Figure 7(b): while only modest effects are present for the smaller systems, strong linear correlation effects are observed for the larger chains with values reaching above 0.8 . The entanglement is monitored through the number of entangled states in Figure 7(c). In the case of the smallest systems, this value is close to one, in agreement with weaker correlation effects. For the larger systems the number of entangled states goes up as the exciton size levels off. There is a steady increase in $Z_{H E}$ indicating that this value would grow further for larger systems. Generally speaking, Figure 7 shows the inter-relations between the exciton size, electron-hole correlation, and entanglement. While the exciton size and correlation coefficient are computed from multipole moment expectation values, ${ }^{13,16}$ the entanglement entropy is computed by an entirely different approach, by analyzing the NTO eigenvalue spectrum. The fact that these values lead to coherent trends underlines the consistency of the theoretical framework employed.

\section{Naphthalene}

To illustrate electron-hole entanglement in a smaller system, naphthalene is studied as a simple small molecule
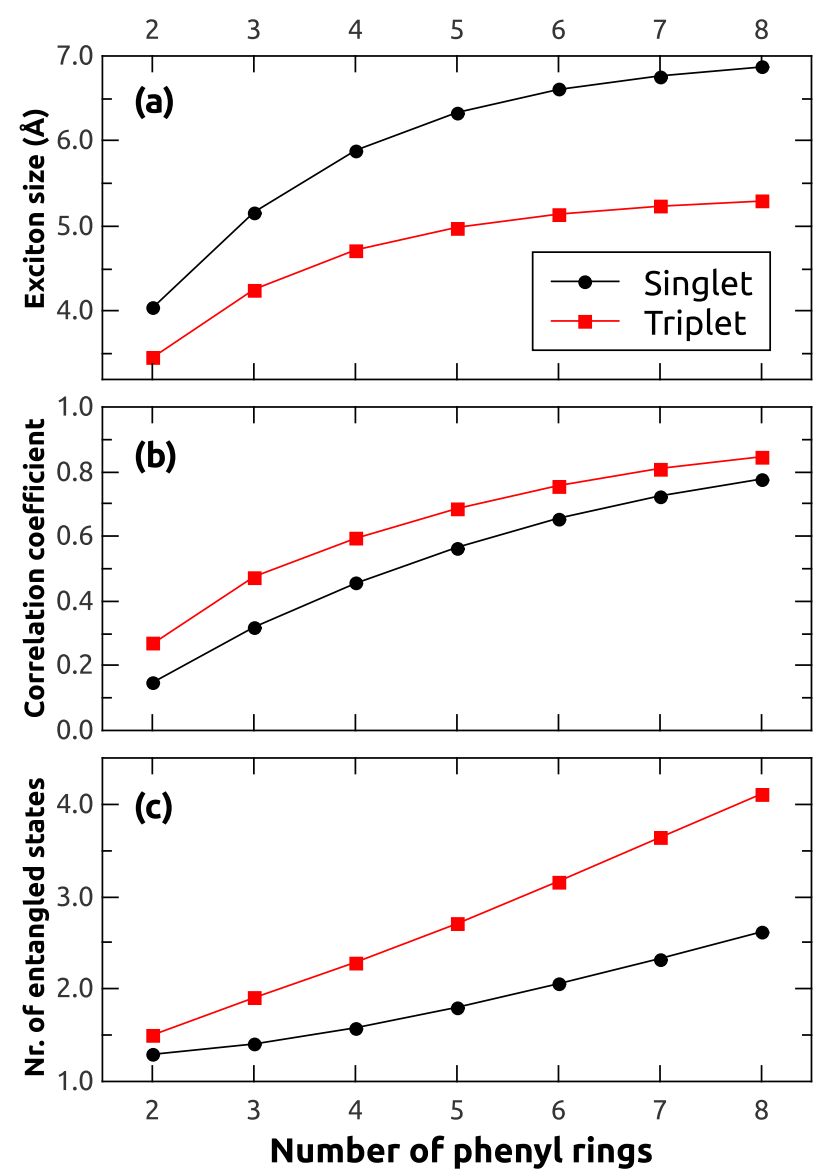

FIG. 7. Analysis of the first singlet and triplet excited states of PPV oligomers of varying size computed at the CAM-B3LYP/SVP level of theory: (a) exciton size $\left(d_{e x c}, \AA\right)$, (b) spatial electron-hole correlation coefficient $\left(R_{e h}\right)$, and (c) number of entangled states $\left(Z_{H E}\right)$.

that is well-characterized computationally. ${ }^{25,59,85,86}$ The lowest excited state is the $1^{1} B_{3 u}$ state, also denoted $L_{b}$, which is characterized by a very low transition strength $(f=0.0001)$ polarized along the long molecular axis. This state is reached by a linear combination of the HOMO-1 to LUMO and HOMO to LUMO+1 transitions both of which possess amplitudes of about $49 \%$. The fact that the $L_{b}$ state is, for the most part, composed of two independent transitions is reflected by $Z_{H E}=2.15$. The next state, denoted $L_{a}$, is of $1^{1} B_{2 u}$ symmetry. It is optically allowed $(\mathrm{f}=0.094)$ with

TABLE I. The first six singlet $\pi \pi^{*}$ excited states of naphthalene computed at the CAM-B3LYP/SVP level of theory: excitation energy $(\Delta E, \mathrm{eV})$, oscillator strength (f), exciton size ( $\left.d_{\text {exc }}, \AA\right)$, electron-hole correlation coefficient $\left(R_{e h}\right)$, number of entangled states $\left(Z_{H E}\right)$, collectivity index $(\kappa)$, and transition in terms of the canonical orbitals.

\begin{tabular}{lccccccc}
\hline \hline & $\Delta E$ & $\mathrm{f}$ & $d_{\text {exc }}$ & \multicolumn{1}{c}{$R_{e h}$} & $Z_{H E}$ & $\kappa$ & Transition $^{\mathrm{a}}$ \\
\hline $1^{1} B_{3 u}\left(L_{b}\right)$ & 4.69 & 0.00 & 3.17 & 0.08 & 2.15 & 2.05 & $\mathrm{H}_{-1} \mathrm{~L}+\mathrm{HL}+1$ \\
$1^{1} B_{2 u}\left(L_{a}\right)$ & 4.96 & 0.09 & 3.40 & -0.03 & 1.68 & 1.33 & $\mathrm{HL}$ \\
$1^{1} B_{1 g}$ & 6.50 & 0.00 & 3.69 & -0.32 & 2.01 & 1.91 & $\mathrm{H}_{-2} \mathrm{~L}+\mathrm{HL}_{+2}$ \\
$2^{1} B_{3 u}$ & 6.74 & 2.06 & 3.24 & 0.05 & 2.74 & 2.24 & $\mathrm{H}_{-1} \mathrm{~L}-\mathrm{HL}_{+1}$ \\
$2^{1} B_{2 u}$ & 6.85 & 0.37 & 3.32 & 0.00 & 2.25 & 1.56 & $\mathrm{H}_{-1} \mathrm{~L}_{+1}$ \\
$2^{1} B_{1 g}$ & 6.99 & 0.00 & 2.61 & 0.35 & 2.76 & 2.15 & $\mathrm{H}_{-2} \mathrm{~L}_{-} \mathrm{HL}_{+2}$ \\
\hline \hline
\end{tabular}

${ }^{\mathrm{a} C a n o n i c a l}$ orbitals involved: H: HOMO, $\mathrm{H}_{-1}$ : HOMO-1, .... 

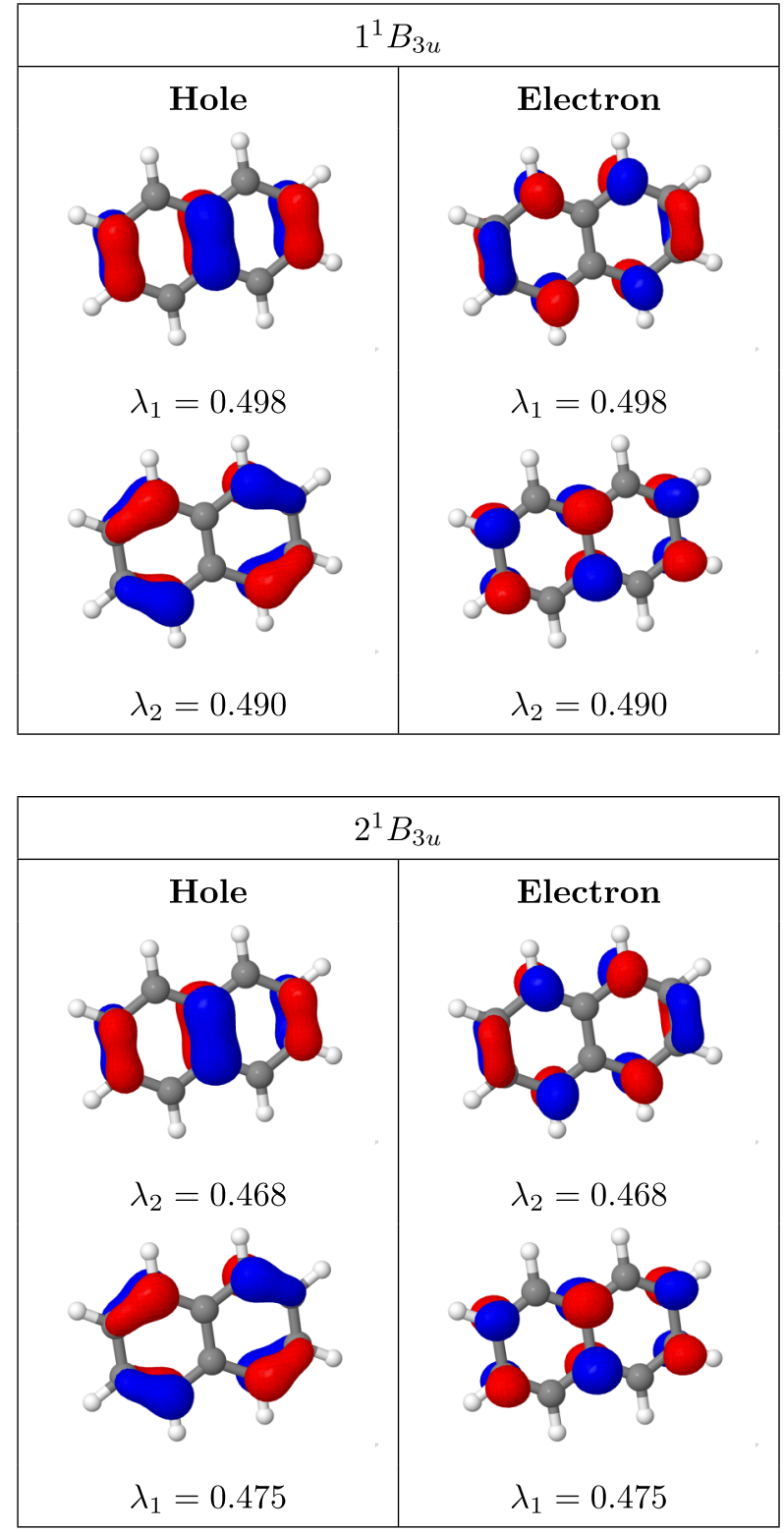

FIG. 8. Natural transition orbitals (isovalue 0.05) of the $1^{1} B_{3 u}\left(L_{b}\right)$ and $2^{1} B_{3 u}$ states of naphthalene, which are virtually indistinguishable, thus illustrating that the decisive differences are encoded in the mutual information between the electron and hole.

polarization along the short axis. This state is dominated by the HOMO-LUMO transition with reduced contributions by other orbitals and thus the number of entangled states is lower, giving $Z_{H E}=1.68$. For all higher states, it holds that $Z_{H E}>2$ reflecting the fact that in all cases several transitions are involved. The collectivity index $\kappa$ (or $\left.\mathrm{PR}_{\mathrm{NTO}}\right)$ as defined in Eq. (15) reflects the trend in $Z_{H E}$ only that $\kappa$ is always somewhat lower. The importance of the mutual information is reflected in Table I. Taking the two ${ }^{1} B_{3 u}$ states as an example, it is observed that these are characterized by almost equivalent orbital transitions considering either the canonical MOs or the NTOs as shown in Figure 8. In other words they are equivalent except for the mutual information between electron and hole, which is represented here by the relative signs of the transitions given in Table I. Nonetheless, dramatic differences are observed with respect to their properties, as seen, e.g., for the excitation energies and oscillator strengths. In the case of the ${ }^{1} B_{1 g}$ states the connection between entanglement and spatial correlation becomes apparent. Despite the fact that both states share the same orbital transitions, the lower energy $1^{1} B_{1 g}$ state shows a negative correlation of $R_{e h}=-0.32$, i.e., effective electron-hole repulsion, whereas a positive correlation of $R_{e h}=0.35$ is obtained for the $2^{1} B_{1 g}$ state. A more detailed phenomenological interpretation of the excited states of naphthalene is out of the scope of this work. However, the important conclusion of the above analysis is that states with identical NTO transitions can possess entirely different properties. Clearly, a standard NTO analysis does not reveal the "whole truth" about an excited state, and some of the crucial properties are determined by superpositions between the individual NTO transitions.

\section{CONCLUSIONS}

In this work it was discussed how the spectrum of the NTO eigenvalues of a one-electron excited state can be used to compute the entanglement entropy between the electron and hole subsystems. In the case of dimers and extended $\pi$-systems this entanglement was related to excitonic correlation effects. The results were compared to our previous spatial exciton analysis protocols and consistent trends were found between the different analysis strategies. Entanglement was also found for the smaller naphthalene molecule and similar effects were previously reported in other areas, e.g., in the context of excimer formation, ${ }^{17}$ crossings between states, ${ }^{17}$ and plasmons. ${ }^{18}$ These effects are related to excited state collectivity ${ }^{7}$ and to the multiconfigurational nature of the excited state but arguably possess a more immediate meaning within the context of quantum information theory. A practical consequence follows from the interpretation of the entanglement entropy $\left(S_{H \mid E}\right)$ as the mutual information between the electron and hole subsystems. If the $S_{H \mid E}$ value is close to zero it suffices to analyze the electron and hole orbitals independently whereas a larger value can serve as a diagnostic to indicate the necessity of more specialized analysis protocols to disentangle correlation and quantum effects.

It is worth noting that correlation and entanglement as discussed here, occur already for CIS, sometimes considered an uncorrelated level of theory. However, CIS clearly creates multiconfigurational wavefunctions in cases where the excited state is not described by a single NTO transition, i.e., the case of $Z_{H E}>1$. It is thus not surprising that correlation effects should surface in such cases, in agreement with a previous analysis. ${ }^{81}$ Enhancing the level of theory to treat dynamic electron correlation affects the results on a quantitative level ${ }^{36,56}$ but does not introduce new qualitative features. An extension to include spin is possible by performing the summation of Eq. (11) over the individual $\alpha$ and $\beta$ eigenvalues and could provide additional information in the case of unrestricted calculations. A more challenging task is the extension of the current formalism to two-electron excitations or to open-shell ground states. 


\section{ACKNOWLEDGMENTS}

The author thanks S. A. Mewes and J.-M. Mewes for comments on the manuscript. This paper is based upon work supported by the VSC Research Center funded by the Austrian Federal Ministry of Science, Research and Economy (bmwfw).

${ }^{1}$ J. Stanton and R. J. Bartlett, J. Chem. Phys. 98, 7029 (1993).

${ }^{2}$ A. Dreuw and M. Head-Gordon, Chem. Rev. 105, 4009 (2005).

${ }^{3}$ M. E. Casida and M. Huix-Rotllant, Annu. Rev. Phys. Chem. 63, 287 (2012).

${ }^{4}$ P. G. Szalay, T. Müller, G. Gidofalvi, H. Lischka, and R. Shepard, Chem. Rev. 112, 108 (2012)

${ }^{5}$ F. Plasser, M. Wormit, and A. Dreuw, J. Chem. Phys. 141, 024106 (2014). ${ }^{6}$ P.-O. Löwdin, Phys. Rev. 97, 1474 (1955).

${ }^{7}$ A. V. Luzanov, A. A. Sukhorukov, and V. E. Umanskii, Theor. Exp. Chem. 10, 354 (1976).

${ }^{8}$ R. L. Martin, J. Chem. Phys. 118, 4775 (2003).

${ }^{9}$ M. Head-Gordon, A. Grana, D. Maurice, and C. White, J. Phys. Chem. 99, 14261 (1995).

${ }^{10}$ S. Tretiak and S. Mukamel, Chem. Rev. 102, 3171 (2002).

${ }^{11}$ A. V. Luzanov, J. Struct. Chem. 43, 711 (2002).

${ }^{12}$ W. Barford and N. Paiboonvorachat, J. Chem. Phys. 129, 164716 (2008).

${ }^{13}$ S. A. Bäppler, F. Plasser, M. Wormit, and A. Dreuw, Phys. Rev. A 90, 052521 (2014).

${ }^{14}$ Y. Li and C. A. Ullrich, J. Chem. Theory Comput. 11, 5838 (2015).

${ }^{15}$ D. Casanova and A. I. Krylov, J. Chem. Phys. 144, 014102 (2016).

${ }^{16}$ F. Plasser, B. Thomitzni, S. A. Bäppler, J. Wenzel, D. R. Rehn, M. Wormit, and A. Dreuw, J. Comput. Chem. 36, 1609 (2015).

${ }^{17}$ F. Plasser and H. Lischka, J. Chem. Theory Comput. 8, 2777 (2012).

${ }^{18}$ T. Yasuike, K. Nobusada, and M. Hayashi, Phys. Rev. A 83, 013201 (2011).

${ }^{19}$ A. V. Luzanov and O. A. Zhikol, Int. J. Quantum Chem. 110, 902 (2010).

${ }^{20}$ K. Sen, R. Crespo-Otero, O. Weingart, W. Thiel, and M. Barbatti, J. Chem. Theory Comput. 9, 533 (2013).

${ }^{21}$ C. A. Guido, P. Cortona, and C. Adamo, J. Chem. Phys. 140, 104101 (2014).

${ }^{22}$ T. Etienne, X. Assfeld, and A. Monari, J. Chem. Theory Comput. 10, 3906 (2014).

${ }^{23}$ S. Matsika, X. Feng, A. V. Luzanov, and A. I. Krylov, J. Phys. Chem. A 118, 11943 (2014).

${ }^{24}$ F. Plasser, S. A. Bäppler, M. Wormit, and A. Dreuw, J. Chem. Phys. 141, 024107 (2014).

${ }^{25}$ E. C. Lim and A. L. L. East, J. Chem. Phys. 113, 8981 (2000).

${ }^{26}$ C. R. Kozak, K. A. Kistler, Z. Lu, and S. Matsika, J. Phys. Chem. B 114, 1674 (2010).

${ }^{27}$ F. Plasser, A. J. A. Aquino, H. Lischka, and D. Nachtigallova, Top. Curr. Chem. 356, 1 (2015).

${ }^{28}$ P. M. Zimmerman, F. Bell, D. Casanova, and M. Head-Gordon, J. Am. Chem. Soc. 133, 19944 (2011).

${ }^{29}$ P. Petelenz and B. Pac, J. Am. Chem. Soc. 135, 17379 (2013).

${ }^{30}$ T. C. Berkelbach, M. S. Hybertsen, and D. R. Reichman, J. Chem. Phys. 141, 074705 (2014).

${ }^{31}$ N. Kirova, Polym. Int. 57, 678 (2008).

${ }^{32}$ C. De Leener, E. Hennebicq, J. C. Sancho-Garcia, and D. Beljonne, J. Phys. Chem. B 113, 1311 (2009).

${ }^{33}$ B. M. Wong and T. H. Hsieh, J. Chem. Theory Comput. 6, 3704 (2010).

${ }^{34}$ R. Binder, J. Wahl, S. Römer, and I. Burghardt, Faraday Discuss. 163, 205 (2013).

35J. Huang, L. Du, D. Hu, and Z. Lan, J. Comput. Chem. 36, 151 (2015).

${ }^{36}$ S. A. Mewes, J.-M. Mewes, F. Plasser, and A. Dreuw, Phys. Chem. Chem. Phys. 18, 2548 (2016).

${ }^{37}$ A. Einstein, B. Podolsky, and N. Rosen, Phys. Rev. 47, 777 (1935).

${ }^{38}$ S. Popescu and D. Rohrlich, Phys. Rev. A 56, R3319 (1997).

${ }^{39}$ J. Audretsch, Entangled Systems: New Directions in Quantum Physics (Wiley-VCH Verlag, 2007).

${ }^{40}$ R. Horodecki, P. Horodecki, M. Horodecki, and K. Horodecki, Rev. Mod. Phys. 81, 865 (2009).

${ }^{41}$ G. K.-L. Chan, Wiley Interdiscip. Rev.: Comput. Mol. Sci. 2, 907 (2012).

${ }^{42}$ K. Boguslawski and P. Tecmer, Int. J. Quantum Chem. 115, 1289 (2015).

${ }^{43}$ Z. Huang and S. Kais, Chem. Phys. Lett. 413, 1 (2005).

${ }^{44}$ K. Pelzer, L. Greenman, G. Gidofalvi, and D. A. Mazziotti, J. Phys. Chem. A 115, 5632 (2011)

${ }^{45}$ G. K.-L. Chan and S. Sharma, Annu. Rev. Phys. Chem. 62, 465 (2011).

${ }^{46} \mathrm{~K}$. Boguslawski, P. Tecmer, O. Legeza, and M. Reiher, J. Phys. Chem. Lett. 3, 3129 (2012)
${ }^{47}$ G. Bester, J. Shumway, and A. Zunger, Phys. Rev. Lett. 93, 047401 (2004).

${ }^{48}$ M. Sarovar, A. Ishizaki, G. R. Fleming, and K. B. Whaley, Nat. Phys. 6, 462 (2010).

${ }^{49}$ A. Thilagam, J. Chem. Phys. 136, 175104 (2012).

${ }^{50}$ F. Troiani, U. Hohenester, and E. Molinari, Phys. Rev. B 62, R2263 (2000).

${ }^{51}$ G. Onida, L. Reining, and A. Rubio, Rev. Mod. Phys. 74, 601 (2002).

${ }^{52}$ S. A. Mewes, F. Plasser, and A. Dreuw, J. Chem. Phys. 143, 171101 (2015).

${ }^{53}$ M. Rohlfing and S. G. Louie, Phys. Rev. B 62, 4927 (2000).

${ }^{54}$ J. Rissler, H. Bässler, F. Gebhard, and P. Schwerdtfeger, Phys. Rev. B 64, 045122 (2001).

${ }^{55}$ S. Klaiman and L. S. Cederbaum, J. Chem. Phys. 141, 194102 (2014).

${ }^{56}$ F. Plasser and A. Dreuw, J. Phys. Chem. A 119, 1023 (2015).

${ }^{57}$ B. Demoulin, M. M. T. El-Tahawy, A. Nenov, M. Garavelli, and T. Le Bahers, Theor. Chem. Acc. 135, 96 (2016).

${ }^{58}$ T. Etienne, H. Gattuso, C. Michaux, A. Monari, X. Assfeld, and E. A. Perpète, Theor. Chem. Acc. 135, 111 (2016).

${ }^{59}$ R. M. Richard and J. M. Herbert, J. Chem. Theory Comput. 7, 1296 (2011).

${ }^{60}$ A. A. Voityuk, Photochem. Photobiol. Sci. 12, 1303 (2013).

${ }^{61}$ I. Mayer, Chem. Phys. Lett. 437, 284 (2007).

${ }^{62}$ P.-Å. Malmqvist and V. Veryazov, Mol. Phys. 110, 2455 (2012).

${ }^{63}$ T. Etienne, J. Chem. Phys. 142, 244103 (2015).

${ }^{64}$ If $m$ is equal to the dimension of the Hilbert space, this is called a maximally entangled state.

${ }^{65}$ The equivalence holds since the $\lambda_{i}$ are the eigenvalues of the $\mathbf{D}^{\mathrm{T}} \mathbf{D}$ matrix and the $\lambda_{i}^{2}$ are the eigenvalues of the $\left(\mathbf{D}^{\mathrm{T}} \mathbf{D}\right)^{2}$ matrix.

${ }^{66}$ Y. Shao, L. F. Molnar, Y. Jung, J. Kussmann, C. Ochsenfeld, S. T. Brown, A. T. Gilbert, L. V. Slipchenko, S. V. Levchenko, D. P. O'Neill, R. A. DiStasio, Jr., R. C. Lochan, T. Wang, G. J. Beran, N. A. Besley, J. M. Herbert, C. Yeh Lin, T. Van Voorhis, S. Hung Chien, A. Sodt, R. P. Steele, V. A. Rassolov, P. E. Malsen, P. P. Korambath, R. D. Adamson, B. Austin, J. Baker, E. F. C. Byrd, H. Dachsel, R. J. Doerksen, A. Dreuw, B. D. Dunietz, A. D. Dutoi, T. R. Furlani, S. R. Gwaltney, A. Heyden, S. Hirata, C.-P. Hsu, G. Kedziora, R. Z. Khalliulin, P. Klunzinger, A. M. Lee, W. Liang, I. Lotan, N. Nair, B. Peters, E. I. Proynov, P. A. Pieniazek, Y. Min Rhee, J. Ritchie, E. Rosta, C. David Sherrill, A. C. Simmonett, J. E. Subotnik, H. Lee Woodcock III, W. Zhang, A. T. Bell, A. K. Chakraborty, D. M. Chipman, F. J. Keil, A. Warshel, W. J. Hehre, H. F. Schaefer III, J. Kong, A. I. Krylov, P. M. Gill, and M. Head-Gordon, Phys. Chem. Chem. Phys. 8, 3172 (2006).

${ }^{67}$ F. Plasser, THEODORE: a package for theoretical density, orbital relaxation, and exciton analysis, 2016, available from http://theodore-qc.sourceforge. net/.

${ }^{68}$ N. M. O’Boyle, A. L. Tenderholt, and K. M. Langner, J. Comput. Chem. 29, 839 (2008)

${ }^{69}$ F. Aquilante, J. Autschbach, R. K. Carlson, L. F. Chibotaru, M. G. Delcey, L. De Vico, I. Fdez Galvan, N. Ferre, L. M. Frutos, L. Gagliardi, M. Garavelli, A. Giussani, C. E. Hoyer, G. Li Manni, H. Lischka, D. Ma, P.-Å. Malmqvist, T. Müller, A. Nenov, M. Olivucci, T. B. Pedersen, D. Peng, F. Plasser, B. Pritchard, M. Reiher, I. Rivalta, I. Schapiro, J. Segarra-Marti, M. Stenrup, D. G. Truhlar, L. Ungur, A. Valentini, S. Vancoillie, V. Veryazov, V. P. Vysotskiy, O. Weingart, F. Zapata, and R. Lindh, J. Comput. Chem. 37, 506 (2016).

${ }^{70}$ A. B. Trofimov and J. Schirmer, J. Phys. B 28, 2299 (1995).

${ }^{71}$ A. Dreuw and M. Wormit, Wiley Interdiscip. Rev.: Comput. Mol. Sci. 5, 82 (2015).

${ }^{72}$ A. Schäfer, H. Horn, and R. Ahlrichs, J. Chem. Phys. 97, 2571 (1992).

${ }^{73}$ T. Yanai, D. P. Tew, and N. C. Handy, Chem. Phys. Lett. 393, 51 (2004).

${ }^{74}$ A. D. Becke, J. Chem. Phys. 98, 5648 (1993).

${ }^{75}$ C. Lee, W. Yang, and R. G. Parr, Phys. Rev. B 37, 785 (1988).

${ }^{76}$ A. N. Panda, F. Plasser, A. J. A. Aquino, I. Burghardt, and H. Lischka, J. Phys. Chem. A 117, 2181 (2013).

${ }^{77}$ S. Hirata and M. Head-Gordon, Chem. Phys. Lett. 314, 291 (1999).

${ }^{78} J_{\text {MOL: }}$ An open-source java viewer for chemical structures in 3D, 2015, available from http://www.jmol.org/.

${ }^{79}$ A. Luzanov and O. Zhikol, in Practical Aspects of Computational Chemistry I, edited by J. Leszczynski and M. K. Shukla (Springer, New York, 2012), pp. 415-449.

${ }^{80}$ I. Mayer, Chem. Phys. Lett. 443, 420 (2007).

${ }^{81}$ P. R. Surjan, Chem. Phys. Lett. 439, 393 (2007).

${ }^{82}$ M. Dallos, H. Lischka, R. Shepard, D. R. Yarkony, and P. G. Szalay, J. Chem. Phys. 120, 7330 (2004).

${ }^{83}$ F. Plasser and H. Lischka, Photochem. Photobiol. Sci. 12, 1440 (2013).

${ }^{84}$ S. Kraner, R. Scholz, F. Plasser, C. Koerner, and K. Leo, J. Chem. Phys. 143, 244905 (2015)

${ }^{85}$ S. Knippenberg, J. H. Starcke, M. Wormit, and A. Dreuw, Mol. Phys. 108, $2801(2010)$

${ }^{86}$ S. Horn, F. Plasser, T. Müller, F. Libisch, J. Burgdörfer, and H. Lischka, Theor. Chem. Acc. 133, 1511 (2014). 